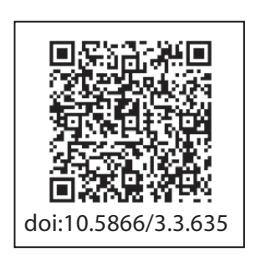

\title{
Lymphangioma of Tongue: A Rare Entity
}

\author{
Supriya M Kheur ${ }^{1}$, Samapika Routray ${ }^{2}$, Yashwant Ingale ${ }^{3}$, Desai R S ${ }^{4}$
}

Reader ${ }^{1}$

Post Graduate Student ${ }^{3}$

Department of Oral Pathology and Microbiology,

Dr.D.Y.Patil Dental College and Hospital,

Pune-18, Maharastra, India.

\section{Senior Lecturer ${ }^{2}$}

Department of Oral Pathology and Microbiology

Geetam Dental College and Hospital

Vishakapattanam, Andhra-Pradesh

Professer, Head of the Department ${ }^{4}$

Department of Oral Pathology and Microbiology,

Nair Dental College \& Hospital,

Mumbai, Maharastra, India

\section{Article Info}

Received: April 15, 2011

Review Completed: May, 16, 2011

Accepted: June, 18, 2011

Available Online: October, 2011

(c) NAD, 2011 - All rights reserved

\begin{abstract}
:
Lymphangiomas are uncommon congenital malformations of lymphatic vessels filled with a clear protein-rich fluid containing few lymph cells. They result from abnormal development of lymphatic system with obstruction to lymph drainage from the affected area. Herewith, we present an interesting case of lymphangioma of the tongue in a 13-year-old boy.
\end{abstract}

Key words: lymphangioma, congenital, malformation

\section{Introduction:}

Lymphangioma is a rare, benign, congenital disease of unknown etiology that originates from lymph vessels and this entity was first described by Virchow in $1854 .{ }^{1}$ It has also been called a malformation, a hamartoma, and an embryonic tumor. Lymphangioma is estimated to make up $6 \%$ of all benign soft tissue tumors in persons younger than 20 years. ${ }^{2}$ Origin of the lesion is considered to be congenital abnormality of the lymphatic system rather than a true neoplasm. It has been postulated that they develop as a result of sequestration of portions of the primitive embryonic lymphatic anlage, failing to anastomoses with the larger lymph channels, therefore functionally existing as localized areas of lymphatic blockage. ${ }^{3}$ Also been argued, instead of being a congenital malformation, lymphangioma is a true neoplasm resulting from transformed lymphatic endothelial cells, stromal cells or both. ${ }^{4}$

Email for correspondence:

drskheur@gmail.com
Lymphangiomas, have a marked predilection for head, neck and oral cavity. ${ }^{3}$ Tongue lymphangiomas typically demonstrate multiple blister like nodules or a pebbly surface that resembles a cluster of translucent vesicles on the enlarged dorsal surface of tongue. ${ }^{4}$ Lymphangiomas are the most common cause of macroglossia in infancy. They are known to be associated with Turner's syndrome, Noonan's syndrome, Trisomies, Cardiac anomalies, Fetal hydrops, Fetal alcohol syndrome, and Familial pterygium colli. Cystic hygroma in a child can grow suddenly due to infection or hemorrhage but can also shrink spontaneously. ${ }^{5}$

Traditionally, they have been divided into four categories based on histologic appearance as-

1. Lymphangioma simplex (capillary lymphangioma, lymphangioma circumscriptum), composed of small, thin-walled, endothelium lined, capillary-sized lymphatic vessels.

2. Cavernous lymphangioma, comprised of dilated lymphatic vessels with surrounding adventitia . 
3. Cystic lymphangioma (cystic hygroma), consisting of huge, macroscopic lymphatic spaces with surrounding fibrovascular tissues and smooth muscle.

4. Benign lymphangioendothelioma (acquired progressive lymphangioma), lymphatic channels appear to be dissecting through dense collagenic bundles. ${ }^{6}$

However, histologic categories do not correlate with clinical behavior or therapeutic response.

So lymphangiomas are also categorized as macrocystic, microcystic, or mixed based on response to sclerotherapy. ${ }^{7}$

\section{Case Report:}

A 13-year-old boy reported to the Department of Oral medicine and Radiology,Dr.D.Y.Patil Dental College and Hospital, Pune with chief complaint of pain and burning sensation in anterior region of the tongue since 8 years. According to the past history, hyperplastic growth was observed on ventral surface of the tongue at the age of 4 years but was left untreated. The lesion gradually increased and is presently involving the entire dorsal surface, ventral surface and lateral border of the tongue.The patient was moderately built and nourished with vital signs within normal limits. He was not in any visible distress at the time of examination. On intraoral inspection, papillary hyperplasia on the dorsal, ventral and lateral border of the tongue was observed which on palpation was soft, slightly tender in nature (Figure 1). Soft tissue ultrasonograph of the tongue demonstrated moderate vascularity on color Doppler studies (Figure 2).

Incisional biopsy under local anaesthesia was performed on the dorsal surface of the tongue and the tissue specimen was sent for histopathological examination. Microscopic section revealed numerous dilated lymphatics lined by endothelial cells filled with lightly eosinophilic proteinaceous material, suggestive of 'lymphangioma.' In many regions the lymph vessels were located just beneath the surface epithelium and exhibited a papillary configuration (Figure 3 \& Figure 4).

Patient was referred to a plastic surgeon for further treatment.

\section{Discussion:}

Lymphangiomas are hamartomatous, congenital malformations of the lymphatic system. There are two theories for the origin of the lymphatic system in humans. One theory is that the lymphatic system develops during the sixth week of embryogenesis from five primitive sacs that arise from the venous system. In the early 1900s, Sabin proposed that in the head and neck, endothelial out pouchings from the jugular sac spread to form the peripheral lymphatic system. Godart, on the other hand, proposed that the lymphatic system developed from mesenchymal clefts in the venous plexus and spread centripetally toward the jugular sac. ${ }^{8}$

Lymphangiomas arise from incomplete development and obstruction of the normal lymphatic system during the sixth week of embryogenesis. Either the primordial lymphatic sacs fail to connect with the venous system. This incomplete canalization of the lymphatics causes obstruction of lymph flow and cyst development. The second theory is that lymph tissues are deposited in the wrong area during embryogenesis and subsequently fail to join the normal lymph system. ${ }^{8}$

In the oral cavity, this lesion is common in the first decade of life and mostly occurs on the dorsal surface and lateral border of the tongue .It rarely arises on palate, gingival, buccal mucosa and lips. ${ }^{1}$ The anterior two-thirds on the dorsal surface of the tongue is the most common site for intraoral lymphangiomas leading to macroglossia. These patients tend to have speech disturbances, poor oral hygiene, and bleeding from the tongue associated with oral trauma. ${ }^{9}$ In our case though the patient has a satisfactory oral hygiene, macroglossia of the tongue is seen with patient complaining of burning sensation as well, suggestive of superimposed infection.

In contrast to hemangiomas, spontaneous regression of lymphangioma is rare and presents as slow, progressive enlargement. ${ }^{1}$ The lymphatic channels dilate with each episode of infection, causing progressive swelling of the tongue. ${ }^{9}$ Lymphangiomas also occur in association with haemangiomas in Maffucci's syndrome. ${ }^{8}$

Fluorescence in situ hybridization techniques can be used to evaluate lymphatic malformations for prenatal chromosomal analysis with emphasis on chromosomes 13,18,21, X, and Y. VEGF-C and its receptors may play an important role in the formation of Lymphatic malformations. ${ }^{7}$

The objectives of treatment of lymphangiomatous macroglossia are preservation of taste, restoration of tongue size for articulation, correction of mandibular and dental deformities, and cosmesis. ${ }^{9}$ The various treatment modalities for lymphangioma are surgical excision, radiation therapy, cryotherapy, electrocautery, sclerotherapy, 
steroid administration, embolization, and ligation, laser surgery with $\mathrm{Nd}-\mathrm{YAG}, \mathrm{CO}_{2} 12$ and radiofrequecncy tissue ablation technique.

Laser photocoagulation has been reported useful in controlling the tongue size and removing superficial lymphangiomata in some cases. ${ }^{3}$

OK-432, a lyphophilized incubation mixture of Strepptoccocus pyogenes and penicillin G potassium has been used to treat lymphangiomas. The sclerosing agent OK-432 is effective for macrocystic lymphatic malformations but showed less promise for microcystic lesions, mixed lesions, and lesions outside the head and neck region. ${ }^{7}$

Histologically, the architecture of blood and lymphatic vessels share similarities but blood vessels recruit supporting pericytes and smooth muscle cells which produce extracellular matrix as the vasculature matures whereas lymphatics have only a sparse covering of recruited supporting cells and lack a continuous basement membrane. Lymphatics have a thin vessel wall and shows positive result with D240 (monoclonal antibody). ${ }^{4}$ Perhaps this discontinuous basement membrane allows the sclerosing agents to permeate freely into the connective tissue, diluting the concentration of the agent and rendering it less effective. ${ }^{3}$

Surgical excision, staged when necessary, continues to be integral to management in many cases. Somnoplasty shows promise for reduction of tongue lymphatic malformations.

\section{References:}

1. Ahadi MMS. Medical Journal of the Iranian Hospital, Dubai, U.A.E.1999;1:34-36.

2. Hartl DM. Extensive Lymphangioma Presenting With Upper Airway Obstruction. Arch Otolaryngol Head Neck Surg 2000;126: 1378-1382.

3. Bernnan TD, Miller AS, Chen S. Lymphangiomas of the oral cavity: A Clinicopathologic, Immunohistochemical and Electron microscopic study. J Oral Maxillofac Surg 1997;55: 932- 935.

4. Yaita T, Onodera K, Xu H, Oya K.Histomorphometrical study in cavernous lymphangioma of the tongue.Oral Disaeses 2007;13:99-104.

5. Jeeva Rathan J et al. Oral lymphangioma: A case report.J Indian Soc Paedod Prev Dent 2005;23: 185-189.

6. Meher R, Garg A, Raj A, Singh I. Lymphangioma Of Tongue. The Internet Journal of Otorhinolaryngology 2005;3 : No2.

7. Bloom DC, Perkins JA and Manning SC .Management of lymphatic malformations. Current Opinion in Otolaryngology \& Head and Neck Surgery 2004;12:500-504.

8. Enzinger FM, Weiss SW. Soft Tissue Tumors. $3^{\text {rd }}$ Edition Mosby; 679-689.

9. Dinerman WS, Myers EN. Lymphangiomatous Macroglossia.The Laryngoscope 1974;291-294.

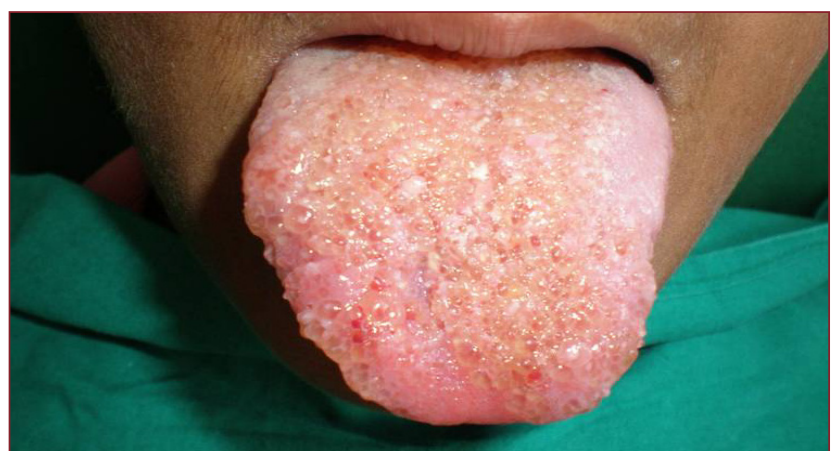

Figure 1. Diffuse lymphangioma of tongue in a 13 year old boy. Note the characteristic appearance of warty looking surface composed of many tiny cysts.

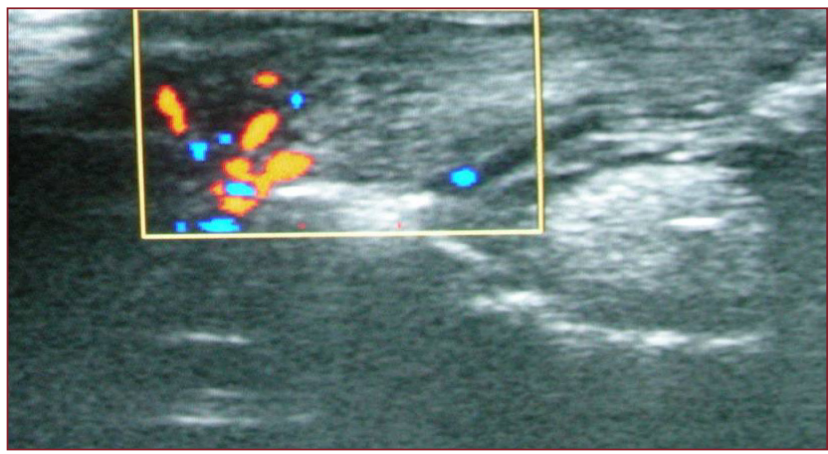

Figure 2. Ultrasonograph of the tongue showing moderate vascularity on color Doppler studies.

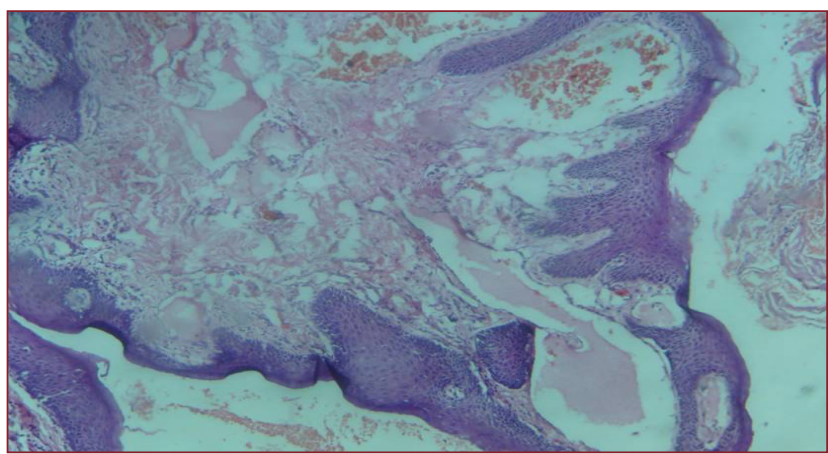

Figure 3. Photomicrograph of lymphangioma showing a papillary surface with engorged lymphatic vessels in the superficial connective tissue( $\mathrm{H} \& \mathrm{E}$, original magnification X10)

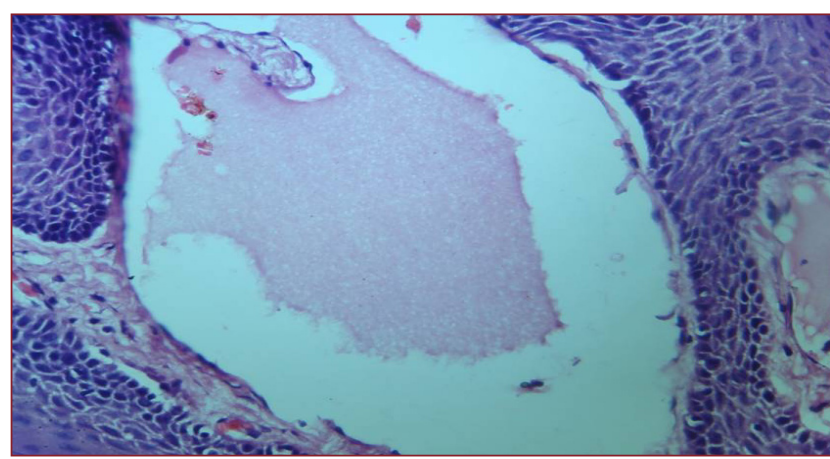

Figure 4. Photomicrograph of lymphangioma showing engorged lymphatic vessels with lymph and thin, discontinuous basement membrane( $H \& E$, original magnification $X 40$ ) 